\title{
Somatisation and function of HPA in fibromyalgia: an investigation using sham and true pain stimuli
}

\author{
Antonella Ciaramella ${ }^{1,2 *}$, Paolo Poli ${ }^{3-5}$ and Marco Maccheroni ${ }^{6}$ \\ ${ }^{1}$ Aplysia onlus, GIFT Institute for Integrative Medicine, Italy \\ ${ }^{2}$ Lecturer in Department of Surgical Pathology, Medical, Molecular and Critical Area, University of Pisa, Italy \\ ${ }^{3}$ Istituto Clinico Villa Aprica, Italy \\ ${ }^{4}$ Casa di Cura San Rossore, Viale delle Cascine, Italy \\ ${ }^{5}$ Consulente del C.I.R.T.A, Università degli Studi di Pisa, Italy \\ ${ }^{6}$ Laboratorio Chimico e di Endocrinologia, Azienda Ospedaliera Universitaria Pisana, Italy
}

\begin{abstract}
Introduction: Studies have shown hypothalamic-pituitary-adrenal (HPA) dysfunction in fibromyalgia, particularly when associated with depression or anxiety Although research has demonstrated a close relationship between fibromyalgia and somatoform disorder, there is as yet no literature on how this association affects HPA activity in fibromyalgia. We set out to investigate the influence of somatoform dimensions on HPA activity using nocebo and cold pressure test (CPT) stimuli.

Method: HPA activity was compared in fibromyalgia patients (n. 20 females) and pain-free controls (n. 14 females) using a one-way between-subjects design. Blood samples were taken at 10:00-10:30 am on the tenth day after the start of menstruation in both groups, and on two consecutive days thereafter, to measure levels of cortisol and ACTH. Nocebo testing and CPT were performed in both groups 30 minutes before this procedure on days 2 and 3, respectively. The Somatosensory Amplification Scale (SSAS), Illness Behaviour Questionnaire, and Somatoform Dissociation Questionnaire 20 were used to investigate somatoform dimensions.
\end{abstract}

Results and conclusions: Fibromyalgia subjects showed a statistically significant blunted cortisol variation after these three stressful stimuli, as compared to controls $(\mathrm{F}=4.41 \mathrm{p}=0.03)$, a difference that was correlated with increased Somatosensory Amplification $(\mathrm{F}=6.77 ; \mathrm{p}=0.01)$.

\section{Introduction}

Fibromyalgia (FM) often accompanies multiple chronic functional symptoms like pain, fatigue, sleep disorders, and irritable bowel syndrome [1]. This spectrum of illness might best be characterized as a condition of generalized sensory amplification of bodily experiences, generally, but not limited to, painful complaints. Although some literature suggests that the presence of numerous comorbid physical symptoms among persons with FM is suggestive of somatisation disorder [2], others propose that they may share underlying pathogenic mechanisms $[3,4]$.

Interestingly, a tendency towards somatic amplification has been linked to a higher incidence of reported adverse side effects of drugs (nocebo effects) [5], and a systematic review has documented high levels of nocebo effects in pharmacological trials conducted on fibromyalgic subjects [6]. Furthermore, altered hypothalamic-pituitary-adrenal (HPA) axis function has been associated with both widespread pain and fibromyalgia $[7,8]$ and patients with $\mathrm{FM}$ have been reported to exhibit HPA axis hypoactivity, with reduced basal plasma cortisol, decreased 24-h urinary free cortisol excretion [8-10], and blunted responses to cortisol-releasing hormone (CRH) [11], hypoglycemichyperinsulinemic clamp [12], and interleukin-6 [13]. However, other authors have shown an increased level of cortisol overall, flattened diurnal cycles of cortisol, and a reduction of cortisol reactivity to acute stress in subjects with FM and rheumatoid arthritis (RA), as compared to healthy subjects [14].
Experiments to investigate the relationship between HPA activity and pain have shown that the former increases after the induction of experimental pain via both true and false (nocebo) pain stimuli [15], and that the HPA axis may be more hyperactive after nocebo than ischemic pain stimulation. In order to shed more light on the issue, we set out to investigate how abnormal illness behaviour and somatic amplification affect HPA function after the application of sham and true pain stimuli in patients with FM and pain-free controls.

\section{Methods}

\section{Design}

A one-way between-subjects design was used to compare fibromyalgia patients and currently pain-free controls in terms of HPA activity. The study was conducted in accordance with the Helsinki Declaration, and all procedures were approved by the local Ethics Committee (n.1413/2002). All subjects provided informed written consent prior to their inclusion, and no subject who agreed to enrol in

Correspondence to: Antonella Ciaramella, Aplysia onlus, GIFT Institute for Integrative Medicine, p.za Cairoli 12, 56127 Pisa, Italy, E-mail: ciarantogift@gmail.com / antonella.ciaramella@medpat.unipi.it

Key words: cortisol, HPA, somatisation, somatosensory amplification, fibromyalgia, nocebo

Received: February 04, 2017; Accepted: February 16, 2017; Published: February 18,2017 
the study received any payment.

Blood samples - one for cortisol and one for ACTH - were taken using a butterfly needle and single catheter for 3 consecutive days ( 3 sessions). The adapter was equipped with a synthetic rubber-coated needle in order to prevent leakage of blood during the tube change in the course of sampling.

Session 1 (baseline) was conducted ten days after the start of menstruation. A blood sample was taken from each subject (both groups) at 10:00-10:30 am, and then a one-hour psychological assessment was carried out. Session 2 was performed one day after the first session, and subjects from both groups were exposed to a sham pain stimulus (nocebo test). A blood sample was then taken between 10:00 and 10:30 am, roughly 30 minutes after the sham pain was administered. The final session, Session 3, was conducted one day after the second session, and subjects from both groups were subjected to the cold pressure test. A blood sample was then taken between 10:00 and 10:30 am, roughly 30 minutes after the pain stimulus was administered.

All sessions were considered as stressful, as even the first baseline measurement involved blood being taken, likely to cause a certain degree of stress in the unaccustomed participant. In the nocebo paradigm, the stressor was the uncertainty and expectation of a painful stimulation, and the nocebo session was conducted before the cold pain session to prevent a pain-conditioning response [16]. The true painful stimulation, cold pressure pain, is associated with a systemic sympathetic activation due to the release of catecholamine [17], and sympathetic activation is the first important mediator of stress [18].

Exclusion criteria: Subjects were excluded if blood samples were taken less than three hours or more than four hours after getting out of bed in each session. Any life-altering events or any changes in normal daily activity during the three-day experiment were also cause for exclusion.

FM subjects were excluded if they exhibited an increase in inflammatory factors (i.e., ESR, CRP, RF, fibrinogen), a variation in protein electrophoresis, or ANA or ENA positivity.

\section{Sample}

Participants with FM were recruited from the Pain Therapy Unit of Santa Chiara Hospital, Pisa, Italy. All patients reported at least a 6-month history of widespread pain, and all met the American College of Rheumatology (ACR) criteria for the diagnosis of primary FM [19]. Patients with FM had laboratory values that were not consistent with an inflammatory process, tested negative for rheumatic disease, and showed no radiological evidence of a cause for their pain.

The control group was recruited from among the students at the University of Pisa. These subjects had been pain free for at least one year, as ascertained by a structured interview regarding their medical history.

All participants included in this study were female (aged 20-45 years) with a regular menstrual cycle. No participants were taking any drug treatments for disease (including pain syndromes), or the contraceptive pill. Subjects were excluded if they had any organic disease (diabetes, cardiac disease, hormonal dysfunction, or hepatic or renal disease).

A structured interview was used to collect demographic and familial data, life events, daily and social activities, as well as their lifetime medical conditions.

\section{Blood sampling}

ACTH: Blood was collected by a venipuncture, avoiding haemolysis, and samples were transported in iced EDTA tubes (vacutainer system), and immersed in an ice bath until separation by controlled temperature centrifuge in max. 30 minutes. After separation, the plasma was processed or frozen immediately. Principle of the analytical procedure: ACTH is assayed by 2-step immunometric chemiluminescent method with washing in an automated analytical system (IMMULITE 2000, Siemens DPC).

Cortisol: Blood was collected by venipuncture, avoiding haemolysis, and samples were transported in a vacutainer and centrifuged for 30 minutes in a controlled temperature centrifuge.

Principle of the analytical procedure: cortisol was assayed by a competitive immunometric method using polarized fluorescence (FPIA method) in an automated analytical system (AxSYM, Abbott).

The temperature of the centrifugation was $4-8^{\circ} \mathrm{C}$.

EDTA was used as an anti-coagulant in samples scheduled for ACTH testing, while activating powder was used in cortisol-testing samples.

\section{Psychophysical experiments}

Sham pain stimulation (nocebo test): The experimental design used was a modified version of the paradigm described by Bayer et al. $[20,21]$. The testing room contained an electronic instrument (sham stimulator) located under a computer to create a stressful setting. Two electrodes were connected to the sham stimulator and attached to the subject supraorbitally and bilaterally. Subjects were told that they would receive an undetectable electrical current that increased in a stepwise fashion, and that the current was "safe but often painful". For each step in current, patients were asked to measure the intensity of pain by pressing a number on the keyboard of the computer.

Five steps, for a total time of $20 \mathrm{~min}$, were foreseen in the experimental setting. Each sham pain stimulus was accompanied by auditory and visual signals, provided by a computer program designed by a collaborator from the National Research Center (CNR). Each step included six subsets in which visual and acoustical signals were administered via the computer monitor.

The experimenter (AC) introduced the expectation of an increase in the intensity of the (sham) pain stimulus by 1) turning the instrument's handle, 2) increasing the acoustic intensity of the signal, and 3) saying the phrase: "We are increasing the intensity of the electric current". At each step the signal was increased by $10 \mathrm{~Hz}$, from $110 \mathrm{~Hz}$ to $160 \mathrm{~Hz}$.

All subjects were instructed to use a mouse to control a pointer on a pain indicator (VAS) displaying easily legible settings between 0 and 15. No pain corresponded to a value of 0 . Pain was to be rated at 10 when aspirin or other medication would be required for similar pain (analgesic threshold). Unbearable pain corresponded to a score of 15. The total score for each step was taken as the sum of the pain scores given for each of the six subsets.

The pain intensity score reported for each step was taken as the pain rating. As no painful stimulus was administered by the sham stimulator, an increase in the pain rating along the steps was taken as conditioned pain (nocebo response).

Cold pressure test: The non-dominant limb was immersed in very cold water $\left(-0.5-2^{\circ} \mathrm{C}\right)$ for a maximum of 240 secs, after temperature 
standardization via limb immersion in water at body temperature (37 $\pm 0.5^{\circ} \mathrm{C}$ ) for 240 secs. The pain threshold was identified as the time from the immersion of the limb in the cold water until the first pain sensation, and the pain tolerance as the time from the immersion of the limb in the cold water until the limb was retracted due to intolerable pain $[22,23]$.

\section{Psychological evaluation}

SSAS (Somatosensory Amplification Scale): Somatosensory amplification refers to the tendency of an individual to experience somatic sensations as intense, noxious, and disturbing. Amplification involves 1) hypervigilance, or heightened attentional focus on bodily sensation, 2) the tendency to select and focus on certain relatively weak and infrequent sensations and, 3) a disposition to react to somatic sensations with affect and cognitions that intensify them and make them more alarming and disturbing. People with this tendency may amplify normal physiological sensations (such as peristalsis or fatigue), the somatic concomitants of affect, and the trivial symptoms of benign infirmities (such as headache or cramp), as well as the symptoms of serious medical disease. In this study, somatosensory amplification was measured using a ten-item self-report SSAS questionnaire. Patients were asked to indicate how much each symptom had bothered them in the preceding $24 \mathrm{hrs}$ using a 5-point scale, with responses ranging from "not at all" to "extremely". A higher global SSAS score indicates greater symptom amplification [24,25].

SDQ-20: The Somatoform Dissociation Questionnaire is a 20item self-report tool, developed by Nijenhuis et al. [26] that is used to measure the intensity of somatoform dissociation. Nijenhuis [27] introduced the term "somatoform dissociation" to designate dissociative symptoms that involve the body and cannot be explained by organic disturbances. Dissociation is a characteristic psychological process related to several disorders, including dissociative disorders (fugue, amnesia and dissociative identity disorders), somatisation disorder (somatisation and conversion disorders), and post-traumatic stress disorder [28].

IBQ: The IBQ (Illness Behaviour Questionnaire, Pilowsky and Spence 1983) investigates abnormal illness behaviour (AIB) by means of scores on 7 scales [29]. Scale 1: General Hypochondriasis: high scores indicate a phobic, anxious concern about health, with some insight. Scale 2: Disease Conviction: high scores indicate a preoccupation with symptoms and the belief that a disease is present, as well as non-response to medical reassurance. Scale 3: Psychological versus Somatic Perception of illness: high scores indicate a perception of the illness in psychological terms, while a low score suggests a tendency to somatise. Scale 4: Affective Inhibition: high scores indicate difficulty in communication of personal, especially negative, feelings to others. Scale 5: Affective Disturbance: high scores indicate the presence of anxiety and depression. Scale 6: Denial: high scores suggest a tendency to deny current life distress and to attribute all problems to physical illness. A low score indicates the presence of life stress not attributed to physical illness. Scale 7: irritability: high scores indicate friction in an interpersonal context.

\section{Statistical analysis}

The data were analysed using Statview Software 5.0 from SAS Data Management. After application of Kolmogorov-Smirnov test, which provides details about the Gaussian distribution of data, $t$ test analysis for unpaired group was applied to compare fibromyalgic to control group. Because the experimental design involved both between- and within-subjects design, statistical analysis was performed applying the ANOVA for repeated measures analysis to compare nocebo, cortisol and ACTH sessions differences between groups. The difference between groups of amount of total variation $(\Delta)$ of nocebo pain perception during steps, and cortisol and ACTH during sessions was analysed with ANOVA and investigated the variability related to psychological dimensions with ANCOVA analysis. Data are presented as mean $( \pm \mathrm{sd})$, and the level of significance was taken as $\mathrm{p}<0.05$.

\section{Results}

\section{Demographic and clinical features}

Twenty FM patients and 14 pain-free subjects participated in this study. A total of 37 subjects were selected initially, but the onset of sleep disorders and life events in the experimental period necessitated the exclusion of 3 subjects ( 2 pain and 1 pain free).

The two groups differed by education (Table 1). In particular, the FM group had a lower level of education than the pain-free control group. According to the IBQ scores, fibromyalgic subjects showed more Disease Conviction and Affective Disturbance than the control group (Table 1).

\section{Pain perception}

Although no differences were found between groups in the pain perception (VAS) evoked by the nocebo stimulus at each step (Table 1), fibromyalgic subjects showed higher increased pain perception during nocebo pain induction with respect to pain-free subjects (Figure 1). The total increase in pain perception during nocebo testing was higher in subjects with fibromyalgia with respect to healthy controls (Table

Table 1. Mean scoring and differences between groups of investigated variables.

\begin{tabular}{|c|c|c|c|c|c|c|c|}
\hline & & \multicolumn{2}{|c|}{$\begin{array}{c}\text { Fibromyalgia } \\
\text { (n. 20) }\end{array}$} & \multicolumn{2}{|c|}{ Control (n.14) } & \multirow[t]{2}{*}{ t value } & \multirow[t]{2}{*}{$\mathbf{p}$} \\
\hline & & $\mathrm{xM}$ & $\mathrm{sD}$ & $\mathrm{xM}$ & $\mathrm{sD}$ & & \\
\hline Age & & 32.10 & 5.44 & 28.57 & 5.52 & 1.84 & 0.07 \\
\hline Education (years) & & 10.90 & 4.68 & 14.42 & 2.34 & 2.59 & 0.01 \\
\hline Onset of FM (months) & & 52.55 & 25.57 & & & & \\
\hline Body Mass Index (BMI) & & 22.53 & 2.58 & 22.25 & 2.77 & 0.30 & 0.76 \\
\hline SSAS & & 17.45 & 6.81 & 14.92 & 5.01 & 1.17 & 0.24 \\
\hline Hypochondriasis & & 2.95 & 1.82 & 1.92 & 1.68 & 1.65 & 0.10 \\
\hline Disease Convinction & & 3.60 & 1.53 & 1.21 & 0.89 & 5.21 & $<0.0001$ \\
\hline Psycho/Somat Focusing & & 2.25 & 1.37 & 2.14 & 1.09 & 0.24 & 0.81 \\
\hline Affective Inhibition & & 2.35 & 1.63 & 1.85 & 1.56 & 0.88 & 0.38 \\
\hline Affective Disturbance & & 3.80 & 1.19 & 1.71 & 1.63 & 4.29 & 0.0002 \\
\hline Denial & & 2.85 & 1.69 & 1.92 & 1.14 & 1.76 & 0.08 \\
\hline Irritability & & 3.00 & 1.48 & 2.50 & 1.65 & 0.92 & 0.36 \\
\hline SDQ & & 4.00 & 3.96 & 2.61 & 2.32 & 1.13 & 0.26 \\
\hline Threshold & & 17.09 & 17.44 & 15.83 & 11.43 & 0.29 & 0.81 \\
\hline Tolerance & & 51.20 & 40.31 & 62.68 & 36.56 & 0.84 & 0.40 \\
\hline \multirow[t]{5}{*}{ nocebo (VAS) } & step 1 & 4.15 & 8.31 & 2.28 & 5.07 & 0.74 & 0.46 \\
\hline & step 2 & 7.00 & 10.99 & 3.85 & 5.69 & 0.17 & 0.33 \\
\hline & step 3 & 10.75 & 14.31 & 4.14 & 5.53 & 1.63 & 0.11 \\
\hline & step 4 & 13.85 & 16.83 & 7.92 & 7.81 & 1.22 & 0.23 \\
\hline & step 5 & 16.65 & 20.34 & 11.64 & 13.46 & 0.80 & 0.42 \\
\hline \multirow[t]{3}{*}{$\operatorname{cortisol}(\mathrm{mcg} / \mathrm{dl})$} & baseline & 11.26 & 4.15 & 13.54 & 8.56 & 1.03 & 0.30 \\
\hline & nocebo & 11.28 & 6.44 & 16.23 & 15.28 & 1.29 & 0.20 \\
\hline & cold & 9.97 & 5.67 & 14.24 & 12.90 & 1.28 & 0.20 \\
\hline \multirow[t]{3}{*}{ ACTH (pg/ml) } & baseline & 10.09 & 4.08 & 11.80 & 4.39 & 1.15 & 0.25 \\
\hline & nocebo & 15.01 & 18.01 & 12.07 & 6.37 & 0.56 & 0.57 \\
\hline & cold & 10.50 & 4.19 & 9.62 & 3.60 & 0.67 & 0.53 \\
\hline
\end{tabular}

$\mathrm{t}: \mathrm{t}$ test analysis 


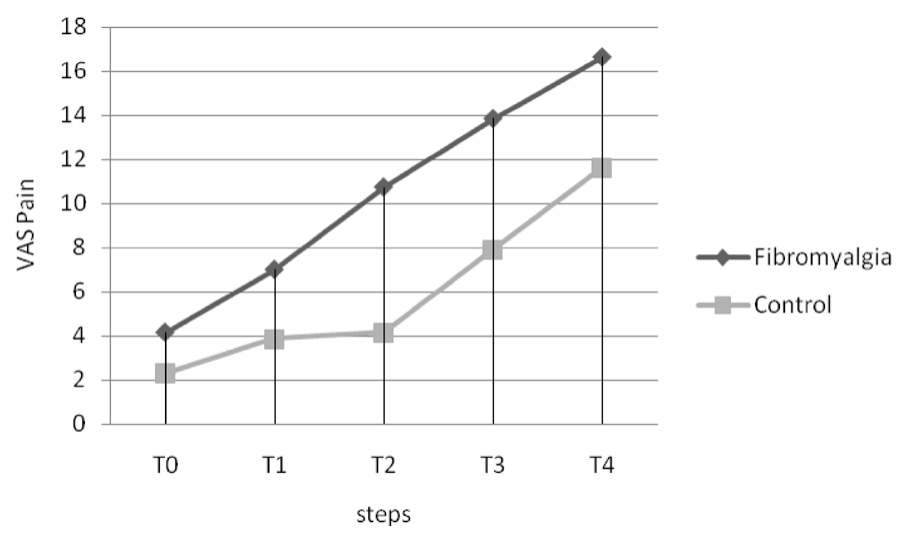

Figure 1. Nocebo paradigm. Five steps, for a total time of $20 \mathrm{~min}$, were foreseen in the experimental setting. Each step comprised six subsets in which visual and acoustical signals were administered via a computer monitor. Both groups showed a greater increase in the perception of pain due sham stimuli.

Table 2. Variation of nocebo, cortisol and ACTH between groups.

\begin{tabular}{|l|c|c|c|c|c|c|c|c|}
\hline & Fibromyalgia & & & Control & & & & \\
\hline & count & $\mathrm{xM}$ & $\mathrm{sD}$ & count & $\mathrm{xM}$ & $\mathrm{sD}$ & $\mathrm{F}$ & $\mathrm{p}$ \\
\hline$\Delta$ Nocebo & 100 & $10.48^{\wedge}$ & 15.17 & 70 & 5.97 & 8.60 & 5.04 & 0.02 \\
\hline$\Delta$ Cortisol & 60 & $10.85 \#$ & 5.44 & 42 & 14.66 & 12.29 & 4.41 & 0.03 \\
\hline$\Delta$ ACTH & 60 & 11.95 & 11.22 & 42 & 11.14 & 4.89 & 0.18 & $\mathrm{~ns}$ \\
\hline
\end{tabular}

ANOVA analysis: $\wedge$ ANCOVA Analysis ( $\triangle$ Nocebo as dependent variable) differences between groups: Affective Disturbance (covariate) $\mathrm{F}=17.85$; $\mathrm{p}<0.0001$; \# ANCOVA Analysis ( $\Delta$ cortisol as dependent variable) difference between groups: SSAS (covariate) $\mathrm{F}=6.77, \mathrm{p}=0.01$.

2). When we have used the variation $(\Delta)$ of Nocebo as a dependent variable, the ANCOVA analysis revealed some different influences of somatisation dimensions (covariates). We showed a difference between groups in the influence of the Affective Disturbance of IBQ on nocebo response $(\mathrm{F}=17.85 ; \mathrm{p}<0.0001)$ (Table 2$)$.

\section{Influences between variables}

No differences were found in the cold pain threshold and tolerance or the induction of the nocebo stimulus between groups. No differences were found in the SSAS, SDQ-20 and IBQ dimensions between the FM and pain-free control groups (Table 1). ANCOVA analysis showed no influence of age on the variations $(\Delta)$ in nocebo response blood cortisol concentration among sessions or blood ACTH concentration among sessions. However, when we used the variation $(\Delta)$ of cortisol or ACTH (during the 3 sessions) as a dependent variable, the ANCOVA analysis revealed some different influences of somatisation dimensions (covariates). The variation of cortisol was shown to be different between the two groups in the presence of an increase of SSAS. The ANCOVA analysis (using $\Delta$ cortisol as the dependent variable) showed a difference between groups in the presence of high levels of SSAS scores (covariate) $\mathrm{F}=6.77 ; \mathrm{p}=0.01$ ) (Table 2).

\section{HPA function}

No differences were found between the FM and control groups in the blood concentration of either cortisol or ACTH at any session (Table 1). However, statistically significantly less variation $(\Delta)$ was found in the plasma concentrations of cortisol after the three stressor stimuli in the FM group with respect to controls (Figure 2). In particular, the fibromyalgic group, showed a reduced cortisol increase with a blunted cortisol response to three different stressful stimuli $(\Delta)$ with respect to the pain-free control group (Table 2). Furthermore, fibromyalgic subjects showed increased levels of blood ACTH after nocebo and cold stimuli, as compared to the control group, although the difference in the variation $(\Delta)$ was not statistically significant (Figure 3 and Table 2 ).

\section{Discussion}

Widespread pain is an essential, but not the only criterion, for the diagnosis of FM. According to the American College of Radiology (ACR) criteria [19] at least 11 tender points are needed for a diagnosis of FM, but new criteria for fibromyalgia have been recently proposed [29]. Indeed, a cross-sectional population study [30] reported a relationship between alterations in HPA function and widespread pain. However, studies exploring the HPA axis in FM have found no clear relationship between the presence of FM and HPA dysfunction [31-33].

We have previously indicated that widespread pain could be associated not only with FM, but also with "masked depression" $[32,34]$, and we set to explore this potential link further. Hence, unlike the great majority of studies in the literature, which rely on daily monitoring of cortisol secretion, we investigated HPA activity after the application acute stressful stimuli, which we considered a good method of exploring the rigidity of the axis which is expressed in the lack of different response (or blunted) to several stressful stimuli $(\Delta)$.

Investigating the response of the HPA axis to stressful stimuli, the only differences we observed between patients with FM and pain-free control subjects was the variation of blood cortisol after all three stressful

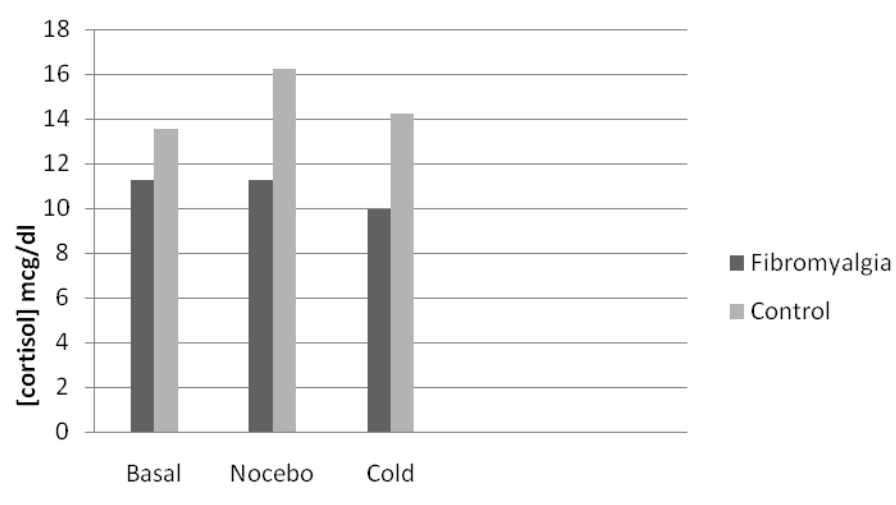

day

Figure 2. Venipuncture was performed at the same time (10.00-10.30 am) on three consecutive days, and blood samples showed a non-statistically significant blunted cortisol variation in response to the different stimuli in fibromyalgic with respect to pain free subjects. ANOVA for repeated measures.

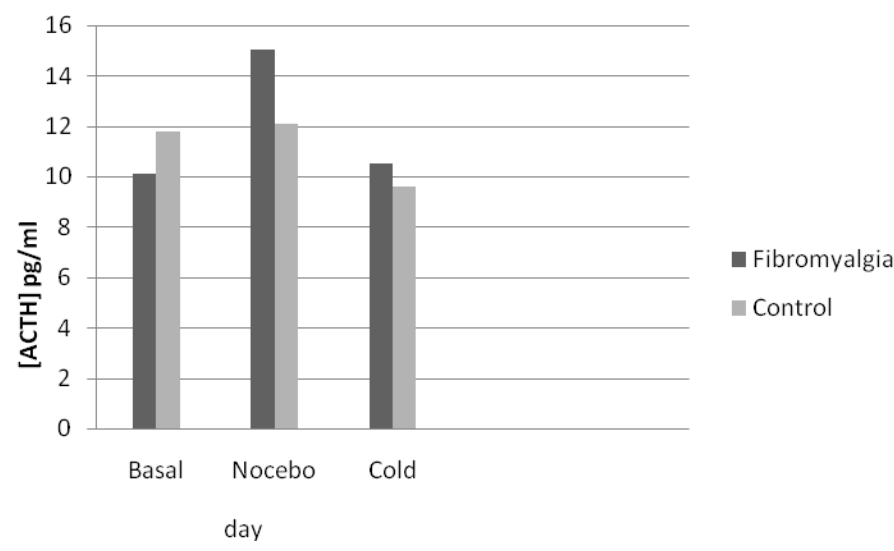

Figure 3. Venipuncture was performed at the same time $(10.00-10.30 \mathrm{am})$ on three consecutive days, and blood samples showed a non-statistically significant variation of ACTH in response to the different stimuli in fibromyalgic with respect to pain free subjects. ANOVA repeated measures. 
conditions (unfamiliar blood test, sham and true pain). Specifically, the FM group showed a smaller variation in cortisol concentration after stressful stimuli, perhaps indicating HPA axis inflexibility in subjects with FM.

However, the fact that we found no statistically significant differences in the variation of blood ACTH between the two groups makes this evidence hard to explain. Nevertheless, some reports have suggest a non-linear relationship between measures of plasma ACTH and blood cortisol [35], and in our FM group the HPA response to three stressful stimuli was expressed in terms of higher levels of ACTH corresponding with lower levels of cortisol with respect to controls. Our cortisol findings were statistically significant when compared to analogous measurements taken in the control group (Table 2), and confirm previous studies indicating HPA axis activity dissociation in FM $[4,5,9-11,36,37]$. In particular, and in accordance with a previous study [36], our results suggest dissociation between the hypothalamicpituitary and adrenal gland axes.

Considering the normal flexibility of HPA system responsiveness, which may produce different blood cortisol and ACTH responses to different stimuli, the blunted response of cortisol seen consistently in the FM group across our experimental sessions would seem to indicate that their HPA activity is compromised by an overstressed system. However the dissociation between ACTH and cortisol plasma concentrations found in our experimental setting, i.e., fibromyalgic subjects showing lower variability in cortisol but higher variability in ACTH than control subjects after three different stressor stimuli, would seem to suggest, as reported by other authors $[10,11]$, some adrenal resistance to ACTH stimulation, which is expressed as a downregulation of ACTH receptors on the adrenal cortex. As previously suggested by other authors [37], this blunted response of the adrenal cortex to ACTH activation after application of pain stimuli could be a function of the adaptive response of the adrenal gland.

Several factors, including depression and anxiety, have been suggested as reasons for HPA axis activity dissociation [38], and several studies have shown a close relationship between somatoform disorders (in which physical symptoms are produced by the mind rather than any identifiable organic cause) and FM. However, there have been very few attempts (if any) to explore the potential relationships between somatosensory amplification and abnormal illness behaviour - both fundamental dimensions of somatoform disorders - and HPA function in fibromyalgic subjects. In fact, to our knowledge, this is one of the first studies to address how the perception of a painful stimulus produced by the mind (sham) can impact on the stress system with respect to a real stimulus in FM.

In our FM group, the HPA axis appeared to respond to stressful stimuli with a lesser secretion of cortisol than that seen in controls. Our results showed that in the FM group, the blunted response to these three stressful stimuli, increases in the presence of high level of SSAS (Table 2).

The blunted cortisol response seen overall in FM was found to be associated with higher scores for SSAS, potentially indicating a role for somatoform dimensions in HPA dysfunction. Our results also suggest that the greater nocebo response seen in subjects with fibromyalgia may be linked to the Affective Disturbance criteria of AIB (Table 2).

Somatosensory amplification is a transversal dimension that may be present in several psychiatric disorders, not only in somatisation disorder [39-41]. Although we found no absolute differences in somatosensory amplification between the FM and control groups, we did note that in the former this dimension was potentially correlated with the lesser variation of HPA activity in response to stress. This supports the assumption that in female subjects an amplifying somatic style may induce a greater susceptibility to stress [42], which is manifested through somatic complaints.

That being said, there are several limitations to our study. In particular, we did not take pre- and post-test blood samples in the respective sessions. However, the fixed time interval between getting out of bed and testing, and the exclusion of subjects with life-altering events or changes in normal daily activity resulted in a relatively controlled design, taking into account the diurnal rhythm of HPA axis activity.

Another limitation of our study was the small number of subjects investigated, which may be responsible for the large variation observed in our report. Nevertheless, as far as we know there has been no larger HPA axis study conducted on FM patients assuming the ACR criteria [19] to confirm the findings reported by McBeth [30], and therefore our results may be of interest. Moreover, our study is one of the first to investigate the respective influences of sham and true pain stimulation on HPA function in fibromyalgic subjects.

\section{Conclusions}

As previously reported [6], our FM subjects exhibited the nocebo phenomenon after sham pain stimulation to a greater extent than pain-free controls (Figure 1). This may be linked to the higher affective disturbance scores that we found in FM subjects (Table 1) having different effects on the nocebo response (Table 2). Furthermore, a blunted cortisol response (Figure 2) was found in the FM group compared pain free control group (Table 2) and not statistically significant increase in blood concentrations of ACTH after stressful stimuli in FM subjects with respect to controls (Figure 3 and Table 2), confirming previous reports of a dissociation of HPA activity in FM [10,11]. Our FM subjects showed higher association between somatosensory amplification and cortisol blunted response to several stressful stimuli (Table 2) that is an expression of HPA axis alteration in the presence of somatisation.

\section{Acknowledgment}

We would to thanks Dr. Daniele Franchi for the implementation of the nocebo test.

\section{References}

1. Yunus MB (2015) Editorial review: an update on central sensitivity syndromes and the issues of nosology and psychobiology. Curr Rheumatol Rev 11: 70-85. [Crossref]

2. Katon WJ (2004) The many faces of depression in primary care. J Gen Intern Med 19: 893-895. [Crossref]

3. Clauw DJ (1995) The pathogenesis of chronic pain and fatigue syndromes, with special reference to fibromyalgia. Med Hypotheses 44: 369-378. [Crossref]

4. Clauw DJ, Chrousos GP (1997) Chronic pain and fatigue syndromes: overlapping clinical and neuroendocrine features and potential pathogenic mechanisms. Neuroimmunomodulation 4: 134-153. [Crossref]

5. Barsky AJ, Borus JF (1999) Functional somatic syndromes. Ann Intern Med 130: 910921. [Crossref]

6. Häuser W, Sarzi-Puttini P, Tölle TR, Wolfe F (2012) Placebo and nocebo responses in randomised controlled trials of drugs applying for approval for fibromyalgia syndrome treatment: systematic review and meta-analysis. Clin Exp Rheumatol 30: 78-87. [Crossref]

7. McFarlane AC (2007) Stress-related musculoskeletal pain. Best Pract Res Clin 
Rheumatol 21: 549-565. [Crossref]

8. Calis M, Gökçe C, Ates F, Ulker S, Izgi HB, et al. (2004) Investigation of the hypothalamo-pituitary-adrenal axis (HPA) by 1 microg ACTH test and metyrapone test in patients with primary fibromyalgia syndrome. J Endocrinol Invest 27: 42-46. [Crossref]

9. Crofford LJ, Pillerner SL, Kalogeras KT, Cash JM, Michelson D, et al. (1994) Hypothalamic-pituitary-adrenal axis perturbations in patients with fibromyalgia. Arthritis Rheum 37: 1583-1592. [Crossref]

10. Griep EN, Boersma JW, Lentjes EG, Prins AP, van der Korst JK, et al. (1998) Function of the hypothalamic-pituitary-adrenal axis in patients with fibromyalgia and low back pain. J Reumatol 25: 1374-1381. [Crossref]

11. Griep EN, Boersma JW, De Kloet ER (1993) Evidence for neuroendocrine disturbance following physical exercise in primary fibromyalgia syndrome. J Musculoskel Pain 1: 217-222.

12. Adler GK, Kinsley BT, Hurwitz S, Mossey CJ, Goldenberg DL (1999) Reduced hypothalamic-pituitary and sympathoadrenal responses to hypoglycemia in women with fibromyalgia syndrome. AM J Med 1999; 106: 534-543. [Crossref]

13. Torpy FJ, Papanicolau DA, Lotsikas AJ, Wilder RL, Chrousos GP, et al. (2000) Responses of the sympathetic nervous system and the hypothalamic-pituitary-adrenal axis to interleukin-6: a pilot study in fibromyalgia. Arthritis Rheum 43: 872-880. [Crossref]

14. Catley D, Kaell AT, Kirschbaum C, Stone AA (2000) A naturalistic evaluation of cortisol secretion in persons with fibromyalgia and rheumatoid arthritis. Arthritis Care Res 13: 51-61. [Crossref]

15. Johansen O, Brox J, Flaten MA (2003) Placebo and Nocebo responses, cortisol, and circulating beta-endorphin. Psychosom Med 65: 786-790. [Crossref]

16. Vierck CJ, Green M, Yezierski RP (2010) Pain as a stressor: effects of prior nociceptive stimulation on escape responding of rats to thermal stimulation. Eur J Pain 14: 11-16. [Crossref]

17. Lowery D, Fillingim RB, Wright RA (2003) Sex differences and incentive effects on perceptual and cardiovascular responses to cold pressor pain. Psychosom Med 65: 284291. [Crossref]

18. Goldstein DS (2003) Catecholamines and stress. Endocr Regul 37: 69-80. [Crossref]

19. Wolfe F, Smythe HA, Yunus MB, Bennett RM, Bombardier C, et al. (1990) The American College of Rheumatology 1990 Criteria for the Classification of Fibromyalgia. Report of the Multicenter Criteria Committee. Arthritis Rheum 33: 160-172. [Crossref]

20. Bayer TL, Baer PE, Early C (1991) Situational and psychophysiological factors in psychologically induced pain. Pain 44: 45-50. [Crossref]

21. Bayer TL Coverdale JH, Chiang E, Bangs M (1998) The role of prior pain experience and expectancy in psychologically and physically induced pain. Pain 74: 327-331. [Crossref]

22. Wolff BB (1984) Methods of testing pain mechanisms in normal man. In: Wall PD, Melzack R edtrs. Tektbook of pain. ( $2^{\text {nd }}$ edn). Edinburgh: Churchill Livingstone: $186-$ 194.

23. Sternberg WF, Bailin D, Grant M, Gracely RH (1998) Competition alters the perception of noxious stimuli in male and female athletes. Pain 76: 231-238. [Crossref]

24. Barsky AJ, Goodson JD, Lane RS, Cleary PD (1988) The amplification of somatic symptoms. Psychosom Med 50: 510-519. [Crossref]
25. Barsky AJ, Wyshak G, Klerman GL (1990) The somatosensory amplification scale and its relationship to hypochondriasis. J Psychiatr Res 24: 323-334. [Crossref]

26. Nijenhuis ER, Spinhoven P, Van Dyck R, Van der Hart O, Vanderlinden J (1996) The development and psychometric characteristics of the Somatoform Dissociation Questionnaire (SDQ-20). J Nerv Ment Dis 184: 688-694. [Crossref]

27. Nijenhuis ER (2000) Somatoform dissociation: major symptoms of dissociative disorders. J Trauma Dissociation 1: 7-32.

28. Espirito Santo HM, Pio-Abreu JL (2007) Dissociative disorders and other psychopathological groups: exploring the differences through the Somatoform Dissociation Questionnaire (SDQ-20). Rev Bras Psiquiatr 29: 354-358.

29. Pilowsky I, Spence ND (1983) Manual for the Illness Behaviour Questionnaire (IBQ) $\left(2^{\text {nd }}\right.$ edn). Adelaide: University of Adelaide.

30. Wolfe F, Clauw DJ, Fitzcharles MA, Goldenberg DL, Häuser W, et al. (2011) Fibromyalgia criteria and severity scales for clinical and epidemiological studies a modification of the ACR Preliminary Diagnostic Criteria for Fibromyalgia. J Rheumatol 38: 1113-1122. [Crossref]

31. McBeth J, Chiu YH, Silman AJ, Ray D, Morriss R, et al. (2005) Hypothalamicpituitary-adrenal stress axis function and the relationship with chronic widespread pain and its antecedents. Arthritis Res Ther 7: R992-R1000. [Crossref]

32. López Ibor JJ (1972) Masked depressions. Br J Psychiatry 120: 245-258. [Crossref]

33. Gur A, Cevik R, Nas K, Colpan L, Sarac S (2004) Cortisol and hypothalamic-pituitarygonadal axis hormones in follicular-phase women with fibromyalgia and chronic fatigue syndrome and effect of depressive symptoms on these hormones. Arth Res Therapy 6: R232-R238. [Crossref]

34. McLean SA, Williams DA, Harris RE, Kop WJ, Groner KH, et al. (2005) Momentary relationship between cortisol secretion and symptoms in patients with fibromyalgia. Arthritis Rheum 52: 3660-3669. [Crossref]

35. Hellhammer DH, Wüst S, Kudielka BM (2009) Salivary cortisol as a biomarker in stress research. Psychoneuroendocrinology 34: 163-171. [Crossref]

36. Griep EN, Boersma JW, De Kloet ER (1993) Altered reactivity of the hypothalamicpituitary-adrenal axis in the primary fibromyalgia syndrome. J Rheumatol 20: 469-474 [Crossref]

37. Geiss A, Rohleder N, Anton F (2012) Evidence for an association between an enhanced reactivity of interleukin-6 levels and reduced glucocorticoid sensitivity in patients with fibromyalgia. Psychoneuroendocrinology 37: 671-684. [Crossref]

38. Hauger RL, Risbrough V, Brauns O, Dautzenberg FM (2006) Corticotropin releasing factor (CRF) receptor signaling in the central nervous system: new molecular targets. CNS Neurol Disord Drug Targets 5: 453-479. [Crossref]

39. Nakao M, Barsky AJ (2007) Clinical application of somatosensory amplification in psychosomatic medicine. Biopsychosoc Med 1: 17 .

40. Duddu V, Isaac MK, Chaturvedi SK (2006) Somatization, somatosensory amplification, attribution styles and illness behaviour: a review. Int Rev Psychiatry 18: 25-33. [Crossref]

41. De Berardis D, Campanella D, Gambi F, La Rovere R, Sepede G, et al. (2007) Alexithymia, fear of bodily sensations, and somatosensory amplification in young outpatients with panic disorder. Psychosomatics 48: 239-246. [Crossref]

42. Nakao M, Tamiya N, Yano E (2005) Gender and somatosensory amplification in relation to perceived work stress and social support in Japanese workers. Women Health 42: 41-54. [Crossref]

Copyright: (C2017 Ciaramella A. This is an open-access article distributed under the terms of the Creative Commons Attribution License, which permits unrestricted use, distribution, and reproduction in any medium, provided the original author and source are credited. 